\title{
UNUSUAL INITIAL AND FINAL STATE EFFECTS IN QUANTUM CHROMODYNAMICS
}

Annual Progress Report

for the period July 15, 1992 - December 18, 1992

Charles A. Nelson

Department of Physics

State University of New York at Binghamton

Binghamton, New York 13902-6000

\section{DISCLAIMER}

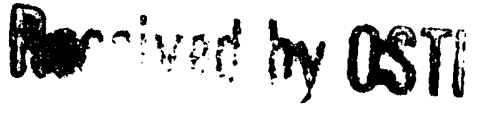

JAN 191993

This report was prepared as an account of work sponsored by an agency of the United States Government. Neither the United States Government nor any agency thereof, nor any of their employees, makes any warranty, express or implied, or assumes any legal liability or responsibility for the accuracy, completeness, or usefulness of any information, apparatus, product, or process disclosed, or represents that its use would not infringe privately owned rights. Reference herein to any specific commercial product, process, or service by trade name, trademark, manufacturer, or otherwise does not necessarily constitute or imply its endorsement, recommendation, or favoring by the United States Government or any agency thereof. The views and opinions of authors expressed herein do not necessarily state or reflect those of the United States Government or any agency thereof.

December 1992

Prepared for

THE U.S. DEPARTMENT OF ENERGY AGREEMENT NO. DE-FG02-86ER40291 


\begin{abstract}
We have constructed a number of fundamental tests which can be used to probe discrete symmetries, and their possible violations, in the required "new physics" beyond the standard model. On-going experiments with unpolarized $\mathrm{e}^{-} \mathrm{e}^{+}$collisions contain many events for the production-decay sequence $\mathrm{e}^{-} \mathrm{e}^{+} \rightarrow \mathrm{Z}^{\mathrm{o}}, \gamma^{*} \rightarrow \tau^{-} \tau^{+} \rightarrow\left(\mathrm{A}^{-} \mathrm{X}\right)\left(\mathrm{B}^{+} \mathrm{X}\right)$. By inclusion of $\rho$ polarimetry observables such experiments enable two distinct tests for leptonic CP violation in $\tau \rightarrow \rho \vee$ decay by generalization of the energy correlation function for $Z^{0}$, or $\gamma^{*} \rightarrow \tau^{-} \tau^{+} \rightarrow$ $\left(\rho^{-} v\right)\left(\rho^{+} \bar{v}\right)$. In other research programs, we are $(i)$ continuing to investigate our proposal that partons be identified with nearly degenerate, coherent quark-gluon "jet" states, and are (ii) investigating the novel consequences of q-analogue quantization of quantum fields, ind of a completeness relation for the q-analogue coherent states.
\end{abstract}


During the past year, a major part of our research effort has been on constructing symmetry tests from generalized spin correlation functions for the production-decay sequence $\mathrm{e}^{-} \mathrm{e}^{+} \rightarrow \mathrm{Z}^{\mathrm{O}}$, $\gamma^{*} \rightarrow \tau^{-} \tau^{+} \rightarrow\left(\mathrm{A}^{-} \mathrm{X}\right)\left(\mathrm{B}^{+} \mathrm{X}\right)$. This has produced the results reported in the publications listed at the end of this "Annual Performance Report." These proposed electroweak tests are particularly relevant to several on-going experiments at the $\mathrm{Z}^{\circ}$ boson at the CERN $\mathrm{e}^{-} \mathrm{e}^{+}$collider LEP and to experiments in the 10-GeV region by the ARGUS and CLEO-II collaborations. Current experiments with unpolarized $\mathrm{e}^{-} \mathrm{e}^{+}$coilisions enable four tests for CP/T violation in the production $\mathrm{Z}^{\mathrm{o}}$, or $\gamma^{*} \rightarrow$ $\tau^{-} \tau^{+}$amplitudes. There are two tests for leptonic CP violation in $\tau \rightarrow \rho v$ decay by inclusion of $\rho$ polarimetry observables in the energy correlation function for $Z^{\circ}$, or $\gamma^{*} \rightarrow \tau^{-} \tau^{+} \rightarrow\left(\rho^{-} v\right)\left(\rho^{+} \bar{v}\right)$. Some of our research time has been spent on other topics, particularly on QCD projects and on the physical implications of the q-analogue harmonic oscillator and of an associated completeness relation for the q-analogue of the usual coherent states.

\section{A. CONSTRUCTION OF FUNDAMENTAL DISCRETE SYMMETRY TESTS FOR NEW PHYSICS}

The $\phi \phi$ parity test proposed by Chang and myself in 1978, as an analogue of C.N. Yang's parity test, was used by the Mark III group at SLAC-SPEAR to experimentally determine the parity of the $\eta_{\mathrm{c}}(2980)$ meson [Phys. Rev. Lett. $\underline{52}, 2126$ (1984)] and to show that the $\omega \omega$ mass distribution peak at about $1.8 \mathrm{GeV}$ in $\mathrm{J} / \psi \rightarrow \gamma \omega \omega$ is predominantly pseudoscalar [Phys. Rev. Lett. 55,1723 (1985)]. The technique was generalized by ourselves in 1984 and by other authors as tool in searching for and establishing the $\mathrm{CP}$ eigenvalue of a technipion/Higgs via the $\mathrm{Z}$ pair or the $\mathrm{W}$ pair decay mode (or the $\tau^{-} \tau^{+}$mode) at a large hadron collider such as the SSC. [J. Phys. (France) Colloq. 46, C2 (1985); Phys. Rev. D33, 93 (1986); Nuc. Phys. B320, 61 (1989)].

In 1984 we also showed that the spin-correlation technique can be used to demonstrate and analyze CP violation in $X \rightarrow S_{1} S_{2}$ sequential decays [Phys. Rev. D30, 1937 (1984)]. Recently 
several physicists have further developed this technique for the study of the $\mathrm{B}^{\circ}$ or $\bar{B}^{\mathrm{o}} \rightarrow \psi+\mathrm{K}_{+}{ }^{*}$ process at a b-fi. tory [See, for example, Dunietz, et.al., Phys. Rev. D43, 2193 (1991)].

In 1989, we extended some of this work to top quark physics. W polarimetry information from $W \rightarrow \bar{\ell} v$ can be used to test for possible CP violation in top quark decay $t \rightarrow W$ b arising from a $\mathrm{t} \overline{\mathrm{t}}$ production process. Examples of useful analogies between $\tau$ polarimetry and $\mathfrak{t}$ polarimetry tests are in C.A. Nelson, Phys. Rev. D41, 2805 (1990).

\section{Tau Production Amplitudes:}

We are currently further developing several techniques for measuring electroweak coupling parameters from beam-referenced $\tau$ spin correlation functions in $\mathrm{e}^{-} \mathrm{e}^{+}$collisions. Previously we showed [Phys. Rev. Lett. 62, 1347 (1989); Phys. Rev. D40, 123 (1989); D41, 2327(E)] that measurement of the energy correlation function $\mathrm{I}\left(\mathrm{E}_{\mathrm{A}}, \mathrm{E}_{\mathrm{B}}\right)$ for $\mathrm{Z}^{\mathrm{O}} \rightarrow \tau_{1}^{-} \tau_{2}{ }^{+} \rightarrow \mathrm{A}^{-} \mathrm{B}^{+} \mathrm{X}$ determines independently the fundamental parameters $\sin ^{2} \theta_{W}$, the tau Michel parameters, and for hadronic tau decays the analogous "chirality parameter" $\xi_{\mathrm{A}}$ which tests for right-handed currents. Similar tests follow for a center of mass energy, e.g. 10 or $4 \mathrm{GeV}$, where an off-shell photon dominates. New information about tau couplings can be obtained by including the direction of the $\mathrm{e}^{-}$beam relative to the final $\mathrm{A}^{-}$and $\mathrm{B}^{+}$momentum directions in the $\mathrm{Z}^{\circ}$ (or $\gamma^{*}$ ) rest frame. In principal, from the resulting "beam-referenced spin-correlation function" (BRSC) the photon and $\mathrm{Z}^{\circ}$ boson couplings, of the tau lepton can be completely measured: A major result is four distinct tests for CP/T violation at the $\mathrm{Z}^{\circ}$ or $\gamma^{*}$ vertex, depending on the value of $E_{\mathrm{cm}}=\downarrow_{\mathrm{s}}$. Nine measurable (slashed/primed) vertex intensity parameters are useful signatures for $\mathrm{CP} / \mathrm{T}$ violation respectively. Should such signature(s) be discovered, vertex intensity relations can be used as a check and to characterize the effect in terms of the polar parametrization of the $Z^{\circ} \rightarrow \tau^{-} \tau^{+}$or $\gamma^{*} \rightarrow \tau^{-} \tau^{+}$helicity amplitudes. The Lorentz structure of these helicity tau production amplitudes is very simple. 


\section{Tau Decay Amplitudes:}

While in $\mathrm{K}^{\circ}$ decay $\mathrm{CP}$ and $\mathrm{T}$ violations are phenomenoligically well-described by the CKM matrix, the fundamental origin of these symmetry violations is still unknown. For this reason it is important to use new collider data to systematically search for possible experimental surprises such as for $\mathrm{CP}$ violation in tau lepton decays. Since the $\rho$ mode has the largest branching ratio, $\mathrm{B}(\tau \rightarrow \rho \mathrm{v}) \approx 22 \%$, on-going experiments with unpolarized $\mathrm{e}^{-} \mathrm{e}^{+}$collisions contain many events for the production-decay sequence.

$$
\mathrm{e}^{-} \mathrm{e}^{+} \rightarrow \mathrm{Z}^{\mathrm{o}}, \gamma^{*} \rightarrow\left(\rho-v_{\tau}\right)\left(\mathrm{B}^{+} \mathrm{X}\right)
$$

where $\mathrm{B}=\rho, \pi, \mu$, e and $\mathrm{X}=\bar{v}_{\tau}$ or $\bar{v}_{\tau} \vee_{\ell}$, and for the CP-conjugate sequence. We find that $\rho$ polarimetry information from the $\rho^{-} \rightarrow \pi^{-} \pi^{0}$ distribution can be quite simply included in the $\tau$ spin-correlation function $\mathrm{I}\left(\mathrm{E}_{\rho}, \mathrm{E}_{\mathrm{B}}\right)$. This yields tests of the CP/T symmetry properties of the helicity amplitudes $A\left(\lambda_{\rho}, \lambda_{v}\right)$ describing $\tau^{-} \rightarrow \rho^{-} v_{\tau}$ and $B(\lambda \bar{\rho}, \lambda \bar{v})$ describing

$$
\tau+\rightarrow \rho+\bar{v}_{\tau}
$$

There are two distinct tests for leptonic CP violation in $\tau \rightarrow \rho \vee$ decay. The two tests are at least ten times more sensitive than the partial width asymmetry

$$
A_{\Gamma} \equiv \frac{\Gamma-\bar{\Gamma}}{\Gamma+\bar{\Gamma}}
$$

For instance, here

$$
\begin{aligned}
& \Gamma=\Gamma\left(\tau^{-} \rightarrow \rho^{-} v\right)=|\mathrm{A}(-1,-1 / 2)|^{2}+|\mathrm{A}(0,-1 / 2)|^{2} \\
& \bar{\Gamma}=\bar{\Gamma}\left(\tau^{+} \rightarrow \rho^{+} \bar{v}\right)=|\mathrm{B}(1,1 / 2)|^{2}+|\mathrm{B}(0,1 / 2)|^{2}
\end{aligned}
$$


The phenomenological significance of the asymmetry signatures also differs: "A $\Gamma$ " tests for possible leptonic CKM-type CP violation. The CP violation observables $\beta_{\mathrm{a}}-\beta_{\mathrm{a}}$ and $\mathrm{r}_{\mathrm{a}} / \mathrm{r}_{\mathrm{b}}$ for $\tau \rightarrow$ $\rho v$ test for other types of CP violation. This is because any overall leptonic-CKM-type phases will equally effect the $A(-1,-1 / 2)$ and $A(0,-1 / 2)$ amplitudes, and so will cancel out in $\beta_{a}$ and $r_{a}$, and similarly for the $\tau^{+} \rightarrow \rho^{+} \bar{v}$ amplitudes.

A masters student, Stephen Goozovat, contributed significantly in various phases of this project over the last three years. Five undergraduate students participated in the research during the spring term and summer of 1991. Their participation was particularly helpful in getting the Monte Carlo simulation KORALB operational and in using it to check our analytic results in the standard model limit. [Two are now in graduate programs in physics (at Penn State and at Cornell); two are currently applying to graduate physics programs.] Both for the research accomplished and for the educational experience of the undergraduates, I would like to be able to continue such undergraduate research participation in future summers. Three masters students are currently contributing to this research program [Ming Yang, Hannah Yang, and Joseph Willie].

\section{B. q-ANALOGUE SYMMETRY STRUCTURES}

Partially as an outgrowth of our work on the usage of coherent quark-gluon "jet" states in QCD, we have investigated some properties of the q-analogue of the usual coherent states. These states can be constructed using the q-oscillator realization of quantum groups. In 1990 with a masters student, Robert Gray, we used q-integration to prove a completeness relation for these states. [Lett. to Editor, J. Phys. A: 22, L945 (1990)].

Joined by another masters student, Alan Chiu, we began investigating the "classical limit" of the q-analogue quantized radiation field. [Phys. Lett. A164, 237 (1992)] The usual quasi-classical coherent states characterize cooperative behavior in the $\mathrm{q}=1$ case so it is natural to use the $|z\rangle_{\mathrm{q}}$ coherent states to investigate properties of the q-analogue quantized field. The q-generalizations of 
the phase operator of Susskind and Glogower (circa 1964), and that of Pegg and Barnett (circa 1988) have been constructed. Both generalizations and associated number-phase uncertainty relations are manifestly $q$-independent in the number basis. But in $|z\rangle_{q}$ basis, the variance of the electric field, $(\triangle E)^{2}$, and the $[Q, P]$ uncertainty relation are increased by a factor $\lambda(z)>1$ where $\lambda(z) \rightarrow$ $\left(q^{-1 / 2}-1\right)|z|^{2}+O(1)$ as $z \rightarrow \infty$ for $0<q<1$. Yet, as $z \rightarrow \infty$ the conventional $\Delta N \Delta \phi_{q}=1 / 2$ and approximate, commutation relation $\left[\mathrm{N}, \phi_{\mathrm{q}}\right]=\mathrm{i}$ of Dirac and follow for the $\mathrm{q}$-analogue quantized field in the $|z\rangle_{\mathrm{q}}$ basis. Physics implications are under investigation.

While this remains, to us, an exploratory program, some further work is warranted because of the mathematical definiteness of the fundarnental ideas, because of the tractability of the necessary analysis, and because of the novel features of the testable physical consequences.

An undergraduate, Mitch Fields, participated in this research program during 1991-1992. Now he is doing graduate work in physics at Yale. This fall, another undergraduate, Michael Gartley, has begun research in this area.

Remarks:

(i) Importance of travel support:

During the past year, the trave! budget has been very useful to our research. We attended and ccnsulted with other physicists at the SSC Conference (U. of Wisconsin); at the Workshop on Harmonic Oscillators (U. of Maryland); at the Second Workshop on Tau Physics (U. of Ohio); at the B Physics Workshop (Cornell U.); at the Colliding Beam Workshop on Top-Quark Physics (U. of Wisconsin); and at the Conference on the Fundamental Aspects of Quantum Theory (U. of South Carolina). In addition, besides our regular visits for theory and experimental seminars at Cornell, we visited Fermilab for about three weeks in July. Associated discussions and electronic-mail exchanges with CLEO-II experimentalists and with LEP experimentalists have been particularly useful to our work on tests for tau lepton couplings. 


\section{(ii) Upgrading of office computer system:}

With funds from the past two year's budgets, I have purchased a 486/33C Gateway 2000, Hewlett-Packard Desk Jet 500 Printer, an Intel FAX/modem board, Mathematica software package MS-DOS 386/7, and Scientific-WORD software. These are all operational in my office. Since October, I have been using the Los Alamos bulletin-board preprint systems. This computer upgrading has taken some of our time this past year, but it is very important for our future research programs.

\section{Time Committed to Project:}

The principal investigator committed $100 \%$ of his time to the project from July 15,1992 through August 3, 1992. From August 24, 1992 until May 17, 1993, he will commit about 40\% of his time. He expects to contribute 100\% of his time from May 17 through July 14, 1993.

\section{Talks Presented:}

1. "Tests for Leptonic CP Violation," SSC Conference, at the University of Wisconsin, April 1992.

2. "Tests for CP Violation in Tau Decays," Second Workshop on Tau Physics, Ohio State University, September 1992 [to appear in the proceedings].

3. "Noval Properties of the q-Analogue Quantized Radiation Field," Workshop on Harmonic Oscillators, University of Maryland, March 1992 [to appear in the proceedings].

4. "Coherence, Correlation and the q-Analogue Quantized Field," poster paper at the conference on the Fundamental Aspects of Quantum Theory, University of South Carolina, December 1992. 
Publications to Date: (3 reprints of each submitted previously to DOE)

1. Tests for CP Violations in Tau Decays, Proceedings of the Second Workshop on Tau Physics, Ohio State University (1992).

2. The q-Analogue Quantized Radiation Field and Its Uncertainty Relations, with S.-H. Chiu and R.W. Gray, Physics Letters A164, 237 (1992).

3. Novel Implicat:ons of the q-Analogue Coherent States, in "Symmetries in Science VI: From the Rotation Group to Quantum Algebras", Plenum Press (1993).

4. Novel Properties of the q-Analogue Quantized Radiation Field, in Proceedings of the Workshop on Harmonic Oscillators, University of Maryland (1992).

Poster Paper: ( 3 copies previously submitted to DOE)

1. Coherence, Correlation and the q-Analogue Quantized Field, at Conference on the Fundamental Aspects of Quantum Theory, University of South Carolina (December, 1992).

Preprints to Date: (1 copy submitted previously to DOE)

1. Tests for Leptonic CP Violation in $\tau \rightarrow \rho \vee$ Decay, submitted to the Physical Review Letters [SUNY BING 7/19/92].

\section{Masters Theses:}

1. Shao-Hsuan Chiu, "The Classical Limit of the q-Analogue Quantized Radiation Field," Masters Thesis, May 1992.

2. Ming-Hsun Yang, "Tests for CP Violation in $\mathrm{t} \rightarrow$ Wb Decay," Masters Thesis (May 1993, expected).

3. Hui-Chun Yang, "Symmetry Tests for $\tau \rightarrow \pi v, \rho v$, and $a_{1} v$ Decay," Masters Thesis (May 1993, expected). 
DATE

FILMED

$2 / 24 / 93$ 
